\title{
The Correlation Between Gravitational Constant and Distance from Matter
}

Abolfazl Soltani *

\author{
Department of Physics, University of Birjand, Birjand, Iran
}

E-mail:soltani.a.physics@gmail.com

\begin{abstract}
Here I show that we are able to consider a large value for the gravitational constant, $G$, at the vicinity of matter $(r<1 \AA)$. Today physics and chemistry extremely need to this choice. By considering a large value for $G$, at the vicinity of matter, the stability of atom and atomic and molecular bonds can be explained by a different method. In this article, I prove that the value of vacuum permittivity, at the vicinity of a charged particle $(r<1 \AA)$, is larger than its present value. Moreover, in this article, it is proved that Spacetime lines interact with electric field lines. Also, here I obtain a new equation (not a new value) for a very important constant, namely the finestructure constant, with the presence of the gravitational constant, $G$. Most of physicists believe that the fine structure constant might hold the clues to the Grand Unified Theory.
\end{abstract}

Keywords: Gravitational Constant, Vacuum Permittivity, Fine-Structure Constant, Spacetime, Electric Field

Introduction: There is gravitational force at the subatomic scale $(r<1 \AA$ ) but its value is very negligible. By considering a large value for $G$, at the subatomic scale, the force of gravity can be increased at this scale, based on $F=G m_{1} m_{2} / r^{2}$. But the presence of a strong gravitational force along with the Coulomb force cause the collapse of the Bohr atom. To avoid this problem, we must choose a different value for $\varepsilon_{0}$, at the subatomic scale. In the following, we will discuss about these issues and show that selecting a large value for $G$ at the atomic scale has very valuable results.

\section{Laboratory Limitations}

There is no laboratory limitations for selecting a large value for $G$ at atomic distances because the value of $G$ at the vicinity $(r<1 \AA$ or $r<1 \mathrm{~nm}$ ) of Proton, Neutron, Electron and nuclei of atoms has not been measured yet. The basis of most of the experiments to measure the value of $G$ is the Newton's law of universal gravitation $\left(F=G m_{1} m_{2} / r^{2}\right)$ and torsion Balance [1]. In these experiments [2-5] the value of $G$ is obtained according to the Newton's law and the known values of $m_{1}, m_{2}, F$ and $r$. In addition, the other various experiments with different methods have been carried out to measure the value of $G$ such as the ones using very cold atoms and atom interferometry [6-9]. Yet, none of these experiments measured the value of $G$ at distances of less than one nanometer or one angstrom $(r<1 \AA$ or $r<1 \mathrm{~nm})$. Therefore, we do not know the value 
of $G$ through experiments, at subatomic distances; and thus we can theoretically consider a large value for it $^{1}$. We have:

$$
\begin{cases}G(r)=G_{0}=6.67 \times 10^{-11} \mathrm{Nm}^{2} \mathrm{Kg}^{-2} & r>1 \AA \\ G(r)=G_{1} \gg G_{0} & r<1 \AA\end{cases}
$$

In the above equations, the boundary between $G_{0}$ and $G_{1}$ (namely $r=1 \AA$ ) is an approximate and selective one, with no emphasis on it.

\section{Physical Concept of Change in the Gravitational Constant}

The presence of an object causes the curvature of Spacetime (Fig. 1). Passing of gravitational waves creates the wrinkles in Spacetime, and variation in the value of $G(r)$ from $G_{0}$ to $G_{1}$ can be considered and imagined as the compression of Spacetime lines. It means that in Fig. 1 the Spacetime lines, at the vicinity of matter $(r<1 \AA)$, compress and the squares become smaller.

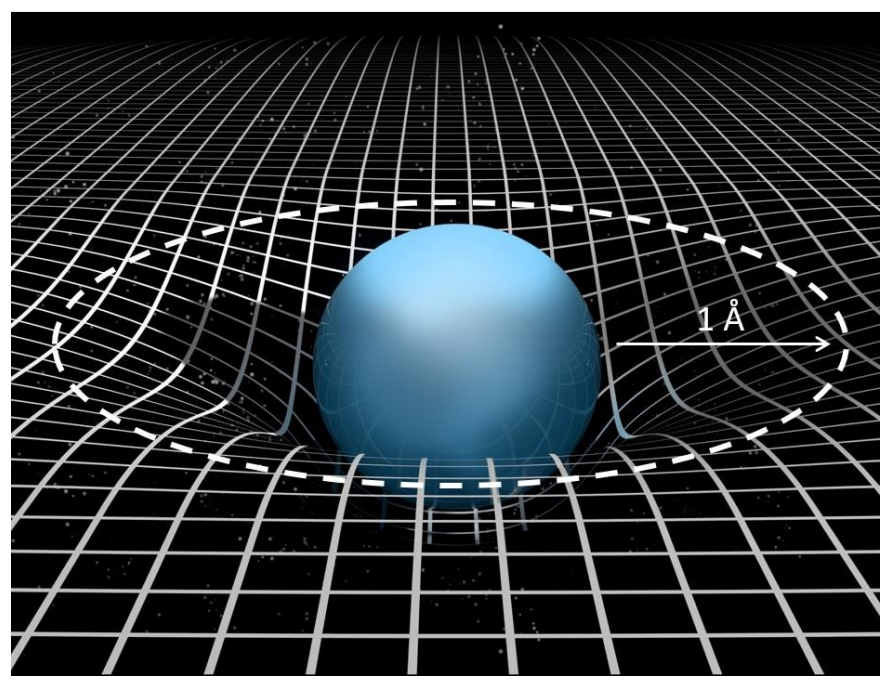

Fig. 1. The presence of an object causes the curvature of Spacetime. The Spacetime lines are shown in this figure. While passing through the boundary of $1 \AA$, and at the vicinity of matter, the spacetime lines compress. and the squares become smaller which this issue is not shown in this figure.

\section{Stability of Atom and Gravitational Effects}

By considering a large value for $G$ at the subatomic scale, the gravitational effect of the atom's nucleus along with the coulomb force must be considered as the reason for the stability of the electrons around the nucleus. Based on this, in this section, we will investigate the simplest atom, namely hydrogen atom. By using the Bohr atomic model we will obtain new equations (not new value) for the radius of the hydrogen atom $\left({ }_{1}^{1} H\right)$, its mechanical energy, the Rydberg constant and the fine-structure constant. First, we need to review the Bohr atomic model.

\footnotetext{
${ }^{1}$ My several investigations and studies have shown that also there is no theoretical barrier to selecting a large value for $G$, at the vicinity of matter $(r<1 \AA$ ). This choice does not create any conflict with any field of physics and we will see that it is in the interest of physics.
} 
In the Bohr model, Bohr assumed that the electron revolves in a circular orbit about the nucleus. and he supposed that the mass of the electron is completely negligible compared to the mass of the nucleus [10] The potential energy for the hydrogen atom is coulomb potential [10][11][12]:

$$
E_{P}(r)=-\int F(r) d r=-\frac{k_{e} e^{2}}{r}
$$

And the centripetal force is:

$$
\frac{k_{e} e^{2}}{r^{2}}=\frac{m_{e} v^{2}}{r} \Rightarrow v=\sqrt{\frac{k_{e} e^{2}}{m_{e} r}}
$$

Where $k_{e}$ is coulomb constant. In the Bohr model, the weak gravitational potential is ignored, so the mechanical energy of the system is [11],[12]:

$$
E=E_{K}+E_{P}=\frac{1}{2} m_{e} v^{2}+\left(-\frac{k_{e} e^{2}}{r}\right) \stackrel{\frac{k_{e} e^{2}}{r^{2}}=\frac{m v^{2}}{r}}{=} E=-\frac{k_{e} e^{2}}{2 r}
$$

In the Bohr model the orbital angular momentum of the electron, $L=m_{e} v r$, must be a constant, because the force acting on the electron is entirely in the radial direction. Applying the Bohr quantization condition to $L[10][11]$, we have

$$
L=n \hbar \Rightarrow m_{e} v r=n \hbar
$$

Therefore, we will obtain the following equations for atomic radius [11],[12]:

$$
\begin{gathered}
m_{e} v r=n \hbar \stackrel{v=\sqrt{\frac{k_{e} e^{2}}{m_{e} r}}}{\Longrightarrow} r_{n}=\frac{n^{2} \hbar^{2}}{k_{e} m_{e} e^{2}} \quad n=1,2,3, \ldots . \\
r_{1}=\frac{\hbar^{2}}{k_{e} m_{e} e^{2}}=0.528 \AA
\end{gathered}
$$

And

$$
r_{n}=n^{2} r_{1}
$$

Where $n$ is the Principal Quantum number. For orbital velocity of electron, we have:

$$
\begin{gathered}
v=\frac{n \hbar}{m_{e} r} \stackrel{(5)}{\Rightarrow} v_{n}=\frac{k_{e} e^{2}}{n \hbar} \\
v_{1}=\frac{k_{e} e^{2}}{\hbar}
\end{gathered}
$$

As you know, the definition of fine-structure constant or Somerfield's constant is the ratio of the speed of electron in the first orbit of the Bohr atom to the velocity of light in vacuum [12]:

$$
\alpha=\frac{v_{1}}{c} \stackrel{(9)}{\Rightarrow} \alpha=\frac{k_{e} e^{2}}{\hbar c}=\frac{1}{137}
$$


In addition, by using the equation 3 we have:

$$
\begin{gathered}
E_{n}=-\frac{k_{e} e^{2}}{2 r_{n}}=-\frac{k_{e} e^{2}}{2 n^{2} r_{1}} \stackrel{(6)}{\Rightarrow} E_{n}=-\frac{k_{e}^{2} e^{4} m_{e}}{2 n^{2} \hbar^{2}} \\
E_{1}=-\frac{k_{e}^{2} e^{4} m_{e}}{2 \hbar^{2}}=-13.5 \mathrm{eV} \\
E_{n}=-\frac{E_{1}}{n^{2}}
\end{gathered}
$$

Based on the Bohr model, the difference between the two energy states in the hydrogen atom is equal to $h v$ :

$$
v=\frac{E_{u p}-E_{\text {low }}}{h} \stackrel{(13)}{\Longrightarrow} v=\frac{E_{1}}{h}\left(\frac{1}{n_{l}^{2}}-\frac{1}{n_{u}^{2}}\right) \stackrel{c=v \lambda}{\Longrightarrow} \frac{1}{\lambda}=\frac{E_{1}}{h c}\left(\frac{1}{n_{l}^{2}}-\frac{1}{n_{u}^{2}}\right)
$$

Where $n_{u}$ and $n_{l}$ are the quantum numbers for the upper and lower energy states, respectively; and $\lambda$ is the wavelength of radiation emitted by the atom[11][12]. The equation 14 is of precisely the same mathematical form as the empirical Rydberg formula, which was derived by Rydberg:

$$
\frac{1}{\lambda}=R\left(\frac{1}{n_{l}^{2}}-\frac{1}{n_{u}^{2}}\right)
$$

Therefore:

$$
R=\frac{E_{1}}{h c} \stackrel{(12)}{\Longrightarrow} R=\frac{k_{e}^{2} e^{4} m_{e}}{4 \pi \hbar^{3} c}=1.0974 \times 10^{-3} \AA^{-1}
$$

Compare this equation with the empirical value of the Rydberg constant namely $1.0968 \times$ $10^{-3} \AA^{-1}$. This empirical value is determined by the precise Spectroscopic Methods [12]. The strong agreement of the empirical value and the theoretical value of the Rydberg constant was a great success for the Bohr model.

Now, we want to do recent calculations by considering a large value for $G\left(G(r)=G_{1}\right)$, at the vicinity of nucleus of hydrogen atom $(r<1 \AA)$. The orbital velocity of the electron is much lower than the velocity of light $v_{1}=\frac{1}{137} c[12]^{2}$. In such a case, the gravitational potential $\left(E_{g P}\right)$ of the hydrogen atom $\left({ }_{1}^{1} H\right)$ is non-relativistic ${ }^{3}$, namely

$$
E_{g P}(r)=-\frac{G_{1} m_{e} m_{p}}{r} \quad r<1 \AA
$$

\footnotetext{
${ }^{2}$ Although, no one has ever measured the velocity of electron in the first orbit of the hydrogen atom with a laboratory device or method; But $v_{1}=\frac{1}{137} c$ (equation 9) is an empirical value. A further explanation is that if equation 9 is not true, the equation 16, which is equal to empirical value of the Rydberg constant, will not be obtained. Therefore, equation 9 is an empirical equation and $v_{1}=\frac{1}{137} c$ is empirical velocity of the electron in the first orbit of the hydrogen atom.

${ }^{3}$ The Lorentz factor $\gamma=1 / \sqrt{1-v^{2} / c^{2}}$ for $v_{1}=\frac{1}{137} c$ is very close to 1 and therefore the relativistic effects are negligible.
} 
Where $m_{e}$ and $m_{p}$ are the mass of electron and proton, respectively. In such a case, the new potential energy $\left(E_{P n e w}\right)$ is equal to the sum of gravitational potential $\left(E_{g P}\right)$ and electrical potential $\left(E_{e P}\right)$ :

$$
E_{\text {Pnew }}=E_{g P}+E_{e P}
$$

It is clear, by considering $E_{\text {Pnew }}$, the values of $m_{e}, m_{p}$ and $G_{1}$ will enter in the recent calculations and the recent equations (such as equations 10 and 16) will have changed; therefore, the theoretical and empirical results will not be equal. To avoid this problem, new potential energy $\left(E_{P n e w}\right)$ should be equal to old potential energy $\left(-\frac{k_{e} e^{2}}{r}\right)$. It means that, we must change the value of Coulomb constant $\left(k_{e}\right)$ at the atomic scale. In such a case, we will have a new value for Coulomb constant (namely $k_{\text {enew }}$ instead of $k_{e}$ ), at the vicinity of electric charge $(r<1 \AA$ ). we have:

$$
E_{\text {Pold }}=E_{\text {Pnew }} \Rightarrow \frac{k_{e} e^{2}}{r}=\frac{k_{\text {enew }} e^{2}}{r}+\frac{G_{1} m_{e} m_{p}}{r} \Rightarrow k_{\text {enew }}=k_{e}-\frac{G_{1} m_{e} m_{p}}{e^{2}}
$$

This is a exciting idea. But, what does it mean? As you know, the definition of Coulomb constant is $1 / 4 \pi \varepsilon_{0}$. Therefore, the variation of Coulomb constant from $k_{e}$ to $k_{\text {enew }}$ means that when we approach to an electric charge, the amount of vacuum permittivity shifts from $\varepsilon_{0}$ to $\varepsilon_{0 n e w}$. This is because of the variation of the gravity constant from $G_{0}$ to $G_{1}$ and the compression of Spacetime lines. It means that the Spacetime lines interact with electric field lines and affect them.

Based on equation 18, we have:

$$
k_{\text {enew }}<k_{e} \stackrel{k_{e}=\frac{1}{4 \pi \varepsilon_{0}}}{=} \varepsilon_{0 \text { new }}>\varepsilon_{0}
$$

In the presence of gravity, the mechanical energy of the hydrogen atom is equal to

$$
E=E_{K}+E_{P}=\frac{1}{2} m_{e} v^{2}+\left(-\frac{k_{\text {enew }} e^{2}}{r}-\frac{G_{1} m_{e} m_{p}}{r}\right)
$$

And the centripetal force is:

$$
\begin{aligned}
& \frac{m_{e} v^{2}}{r}=\frac{k_{\text {enew }} e^{2}}{r^{2}}+\frac{G_{1} m_{e} m_{p}}{r^{2}} \\
& v=\sqrt{\frac{k_{\text {enew }} e^{2}+G_{1} m_{e} m_{p}}{m_{e} r}}
\end{aligned}
$$

By putting the equation 22 in equation 20, we have:

$$
E=\frac{-\left(k_{\text {enew }} e^{2}+G_{1} m_{e} m_{p}\right)}{2 r}
$$

Compare this equation with equation 3 . For atomic radius we have:

$$
m_{e} v r_{n}=n \hbar \Rightarrow r_{n}=\frac{n \hbar}{m_{e} v} \stackrel{(22)}{\Longrightarrow} r_{n}=\frac{n^{2} \hbar^{2}}{m_{e}\left(k_{\text {enew }} e^{2}+G_{1} m_{e} m_{p}\right)} \quad n=1,2,3, \ldots
$$




$$
\frac{1}{\lambda}=\frac{E_{1 \text { new }}}{h c}\left(\frac{1}{n_{l}^{2}}-\frac{1}{n_{u}^{2}}\right) \Longrightarrow R_{\text {new }}=\frac{E_{1 \text { new }}}{h c} \stackrel{(27)}{\Longrightarrow} R_{\text {new }}=\frac{k_{\text {enew }}^{2} e^{4} m_{e}}{4 \pi \hbar^{3} c}
$$

Where $R_{\text {new }}$ is the new equation of the Rydberg constant. Compare equation 28 with equation 16. This new value of Rydberg constant is equal to its recent value $\left(1.0974 \times 10^{-3} \AA^{-1}\right)$ because of using equation 18. Addition to equation 22 the orbital velocity of electron is:

$$
m_{e} v r=n \hbar \stackrel{(24)}{\Longrightarrow} v_{n}=\frac{\left(k_{\text {enew }} e^{2}+G_{1} m_{e} m_{p}\right)}{n \hbar}
$$

And

$$
\alpha_{n e w}=\frac{v_{1}}{c}=\frac{\left(k_{\text {enew }} e^{2}+G_{1} m_{e} m_{p}\right)}{c \hbar}
$$

This is the new equation of the fine-structure constant. Compare this equation with equation 10. According to equation 18, the value of $\alpha_{n e w}$ is the same value as $\alpha(1 / 137)$. In the new equation of the fine-structure constant, $\alpha_{n e w}$, Special relativity (c), electromagnetism ( $k_{\text {enew }}$ and $e$ ), Quantum mechanics $(\hbar)$ and General relativity $\left(G_{1}\right.$ and $\left.m\right)$ are unified.

Eventually, based on all of these discussions, the Coulomb force between the two charged particles like two electrons is equal to

$$
\begin{array}{ll}
F=\frac{k_{e} e^{2}}{r^{2}}=\frac{1}{4 \pi \varepsilon_{0}} \frac{e^{2}}{r^{2}} & r>1 \AA \\
F=\frac{k_{\text {enew }} e^{2}}{r^{2}}=\frac{1}{4 \pi \varepsilon_{\text {onew }}} \frac{e^{2}}{r^{2}} & r<1 \AA
\end{array}
$$

\section{Atomic and Molecular Bonds}

In this article, we showed that a large value for $G$ can be considered at the atomic scale. Based on this, in this section, we will investigate how the hydrogen molecule is composed of two Hydrogen atoms. Consider two hydrogen atoms $\left({ }_{1}^{1} H\right)$ collide with each other. Because of the strong gravitational force between the two electrons $\left(F=G_{1} m_{e}^{2} / r^{2}\right)$, the electrons of the two hydrogen atoms will be attracted to each other (Fig. 2). In such a case, the distance $(r)$ between the two electrons gradually narrows; and the Coulomb force $\left(F=k_{\text {enew }} e^{2} / r^{2}\right)$ gradually increases. Thus, the two electrons will approach together until the attraction gravitational force equals the repulsion 
Coulomb force. In this way, the bond between the two atoms of hydrogen is formed and the other and the electrons. But, the electron-electron interaction is the most effective interaction in a
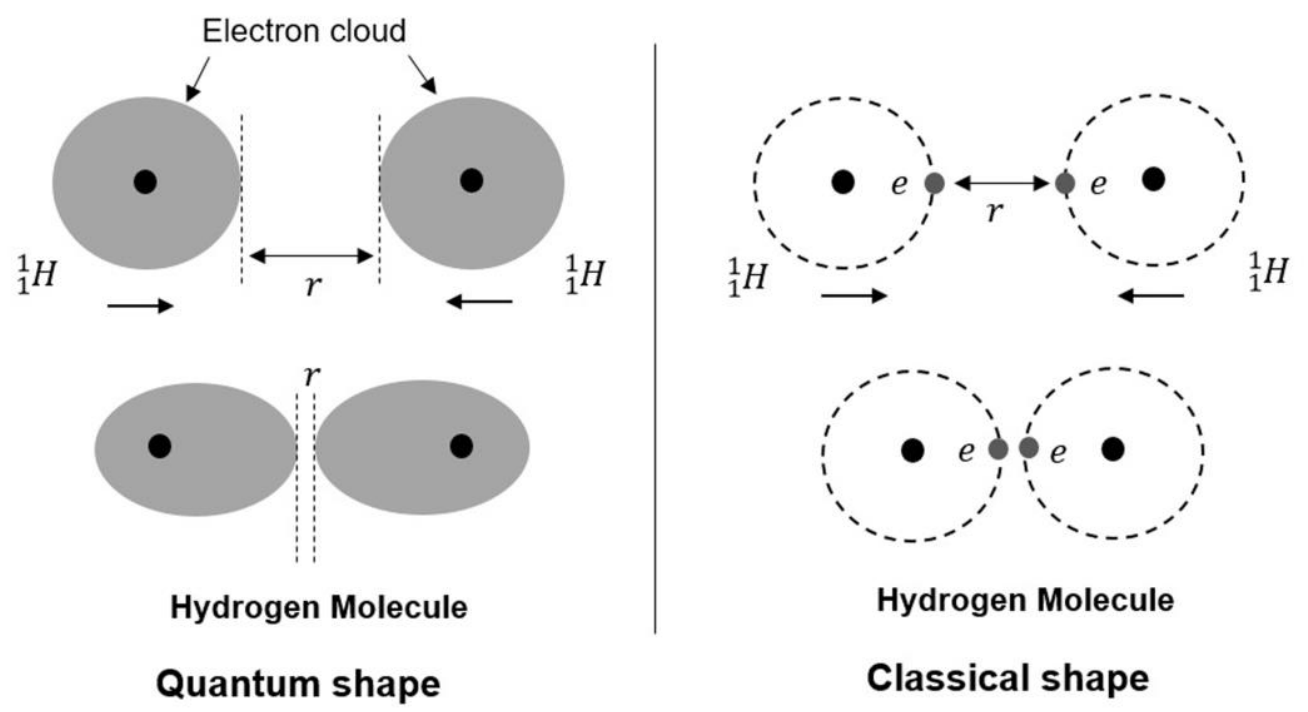

Fig. 2. Hydrogen molecule formation. The two hydrogen atoms are attracted to each other and formed a bond. In the hydrogen molecule, the repulsion Coulomb force between the two electrons is equal to the attraction Gravitational force, based on this article. In the hydrogen molecule and in Quantum shape, the electron clouds of two atoms does not overlap and interference because of the repulsion Coulomb force between the two electrons. therefore, we do not have molecular orbitals in my theory.

By using this description about the hydrogen molecule formation, it is easy to explain other atomic and molecular bonds. In the bonds between atoms and molecules with multiple electrons, several attraction and repulsion forces (arising from the electrons of inner and outer shells of atoms and nuclei) are involved in the formation of bonds and the magnitude of Bond-dissociation energy. For example, in the $\mathrm{F}-\mathrm{H}$ bond, the electron of the hydrogen atom interacts with nine electrons of Fluorine atom and therefore, the bond energy of $\mathrm{F}-\mathrm{H}$ is different from $\mathrm{H}-\mathrm{H}$. For this reason, the energy required to break the $\mathrm{F}-\mathrm{H}$ bond is different from the $\mathrm{H}-\mathrm{H}$ bond ${ }^{4}$. All atomic and molecular bonds in nature can be justified by this description. Here and in atomic bonds and molecular bonds, the gravitational interactions and Coulomb interactions between the electron-electron, electronnucleus and nucleus-nucleus, depending on the distance between the two particles, can be explained through $G_{1}$ and $K_{\text {enew }}$ or $G_{0}$ and $K_{e}$. In other words, in atomic bonds and molecular bonds, the particles which are close to each other $(r<1 \AA$ ), interact with each other with constants $G_{1}$ and $K_{\text {enew }}$ and the particles which are at a distance interacts with each other with constants $G_{0}$

\footnotetext{
${ }^{4}$ As mentioned previously, the electrons also interact with the nuclei, but the main player is the electron-electron interaction, and especially the interaction of the electrons of the outermost shells with each other
} 
and $K_{e}$. The force of gravity along with the Coulomb force can explain all the atomic and molecular bonds in the nature, by a different method.

My theory about atomic and molecular bonds does not conflict with quantum mechanics. In my theory, the wave function of the electrons of the atoms in a molecule does not overlap and interference; and therefore molecular orbitals are not formed.

\section{Conclusion}

In this article, we proved that considering a large value for $G$, at the vicinity of matter, is a very appropriate choice for physics and chemistry. Prior to this article, we thought the contribution of gravity force, at the atomic scale, is negligible. This article revealed that Spacetime lines interact with electric field lines. It is logical that the compression of the spacetime lines affects the electric field lines and the vacuum permittivity. The discovery of interaction of spacetime with electric field can be a way to get to know better the nature of electric field, electric charge and also magnetic field. Moreover, in this article, we obtained new equations for the Rydberg constant and the fine-structure constant. The results and discussions of this article are the beginning of an important change in physics.

\section{References:}

1. Quinn, T. Measuring big G. Nature 408, 919-921 (2000)

2. Cavendish, H. Experiments to determine the density of the Earth. Philosophical Transactions of the Royal Society of London 88, 469-526 (1798).

3. Gundlach, J. Markowitz, S. Measurement of Newton's Constant using a Torsion Balance with angular acceleration feedback. Phys. Rev. Lett. 85(14):2869-72 (November 2000).

4. Luo, J. Liu, Q. Tu, L. Determination of the Newtonian Gravitational Constant G with Time-of-Swing Method. Phys. Rev. Lett. 102, 240801 (16 June 2009).

5. Armstrong, T. Fitzgerald, M. New Measurements of G Using the Measurement Standards Laboratory Torsion Balance. Phys. Rev. Lett. 91, 201101 (13 November 2003).

6. Rosi, G. Sorrentino, F. Cacciapuoti, L. Prevedelli, M. \& Tino, G. Precision measurement of the Newtonian gravitational constant using cold atoms. Nature 510, 518-521 (26 June 2014).

7. Fixler, J. B. Foster, G. T. McGuirk, J. M. \& Kassevich, M. Atom Interferometer Measurement of the Newtonian Constant of Gravity. Science 315, 74-77 (2007)

8. Bertoldi, A. et al. Atom interferometry gravity-gradiometer for the determination of the Newtonian gravitational constant G. Eur. Phys. J. D 40, 271-279 (2006).

9. Lamporesi, G. Bertoldi, A. Cacciapuoti, L. Prevedelli, M. \& Tino, G. M. Determination of the Newtonian gravitational constant using atom interferometry. Phys. Rev. Lett. 100, 050801 (2008).

10. Bohr, N. On the Constitution of Atoms and Molecules. Philos. Mag. 26, 1, (1913)

11. Eisberg, R. Quantum Physics. (John Wiley \& Sons Inc, ed. 2, 1974), pp. 98-107

12. Weidner, R. \& Sells, R. Elementary Modern Physics (Allyn and Bacon, ed. 2, 1973), pp 206-216 